\title{
Optimal Power Allocation for Channel Estimation in MIMO-OFCDM Systems
}

\author{
Yiqing Zhou and Tung-Sang Ng \\ Dept. of Electrical and Electronic Engineering \\ University of Hong Kong, Hong Kong
}

\begin{abstract}
This paper investigates the power allocation in orthogonal frequency and code division multiplexing (OFCDM) systems with multiple input multiple output (MIMO) multiplexing (MIMO-OFCDM). Since time-multiplexed pilot channels are employed for channel estimation, the total transmission power should be properly assigned to the pilot and data channels to achieve the best performance. By minimizing the mean square error (MSE) during signal detection, a closed form expression is derived for the optimal power ratio between the pilot and data channels. It is shown that the optimal power ratio is mainly decided by the number of transmit antennas and the number of pilot and data symbols in a packet, while it is not sensitive to other system parameters like signal-to-noise ratio (SNR) and the frequency domain spreading factor. Simulations demonstrate the accuracy of the derived optimal power ratio.
\end{abstract}

\section{INTRODUCTION}

Orthogonal frequency and code division multiplexing (OFCDM) is a combination of OFDM and two-dimensional (2-D) spreading [1], where each data symbol is spread with orthogonal variable spreading factor (OVSF) codes in the time domain with $N_{T}$ chips and in the frequency domain with $N_{F}$ chips. In OFCDM systems, multiple 2-D code channels can be assigned to one user to increase the downlink transmission rate. However, to achieve a data rate such as $1 \mathrm{Gbps}$ using a bandwidth of $100 \mathrm{MHz}$, multiple-input and multiple-output (MIMO) multiplexing must be considered in the OFCDM system [2].

In practice, the total transmission power is limited and should be properly allocated between pilot and data channels to achieve good performance. Therefore, it is interesting to investigate the power assignment in MIMO-OFCDM systems. Considering time-multiplexed pilot channels, the best system performance can be achieved by using the optimal power ratio between the pilot and all data channels. Furthermore, a joint iterative detection is considered [3], where iterative multi-antenna interference (MAI) cancellation is employed in the space domain to combat co-channel signals from other transmit antennas, and hybrid multi-code interference (MCI) cancellation and minimum mean square error (MMSE) detection [4] is used in the frequency domain to suppress the MCI from other code channels. By minimizing the mean square error (MSE) during the signal detection, a close form expression for the optimal power ratio between the pilot and all data channels $(\beta / K)$ is derived to achieve the best system performance and verified by simulations.

The rest of the paper is organized as follows. Section II introduces the basic structure of the MIMO-OFCDM system. Section III presents a close form expression for the optimal power ratio. Then simulation results are shown in Section IV to verify the derived optimal power ratio. Finally, conclusions are drawn in the last section.

\section{SYSTEM MODEL}

\section{A. Transmitter}

As shown in Fig. 1(a), at the transmitter of the MIMOOFCDM system, information bit streams are firstly serial to parallel (S/P) converted into $n_{t}$ sub-streams, which is further S/P converted into $K$ streams, corresponding to $K$ data code channels. On each code channel, information bits are modulated and $\mathrm{S} / \mathrm{P}$ converted into $M / N_{F}$ symbol streams, where $M$ is the total number of sub-carriers in the OFCDM system. Then, 2-D spreading is carried out, where $N=N_{T} \mathrm{x} N_{F}$ chips can be obtained for each data symbol [4]. The spread signals from all $K$ data code channels are added together at the code multiplexer. The resultant signals pass through a chip interleaver in the frequency domain, so that the $N_{F}$ subcarriers corresponding to the $N_{F}$ chips carrying the same data symbol are separated in the frequency band, providing large frequency diversity gain. At the same time, time multiplexed pilot channels are employed for channel estimation, as shown in Fig. 1(b). Assume that $N_{d}$ and $N_{P}$ are the total number of OFCDM symbols and the number of pilot symbols in one packet, respectively. Choosing $N_{P} \geq n_{t}$, the pilot channels on $n_{t}$ transmit antennas can be spread with different OVSF codes and thus orthogonal to each other. After spreading, the pilot signals pass through the time multiplexer with data signals. The resultant signals are further processed by the IFFT, the guard interval insertion, and the pulse shaping filter. The signal transmitted on the $p^{\text {th }}\left(0 \leq p \leq n_{t}-1\right)$ antenna in one packet duration is given by

$S_{p}(t)=\left\{\begin{array}{l}\sum_{i=0}^{N_{P}-1} \sum_{m=0}^{M-1} d_{p i l o t, p, m} c_{N_{P}, i}^{(p)} e^{j 2 \pi m\left(t-T_{g}-i T_{s}\right) / T_{e}} p\left(t-T_{g}-i T_{s}\right) \\ \sum_{i=N_{P}}^{N_{d}-1} \sum_{m=0}^{M-1} \sum_{k=0}^{K-1} d_{p, i, m, k} C_{i, m, k} e^{j 2 \pi m\left(t-T_{g}-i T_{s}\right) / T_{e}} p\left(t-T_{g}-i T_{s}\right)\end{array}\right.$

where $d_{\text {pilot, }, m}$ is the known pilot symbol with a power of $E\left\{\left|d_{\text {pilot }, p, m}\right|^{2}\right\}=\beta P_{d}, c_{N_{P}, i}^{(p)}$ is the $i^{\text {th }}$ chip of the $p^{\text {th }}$ OVSF code with length $N_{P}, d_{p, i, m, k}$ is the data symbol of the $k^{\text {th }}$ code channel on the $m^{\text {th }}$ sub-carrier in the $i^{\text {th }}$ OFCDM symbol duration with a power of $E\left\{\left|d_{p, i, m, k}\right|^{2}\right\}=P_{d}, C_{i, m, k}$ stands for the 2-D spreading code, $T_{s}=T_{e}+T_{g}$ is the complete OFCDM symbol duration with $T_{e}$ and $T_{g}$ denoting the effective 
OFCDM symbol duration and guard interval, respectively, and $p(t)$ is the pulse shaping filter.

\section{B. Channel Model}

Consider a slow fading parallel multi-channel model. Let $H_{p, q, m}$ be the complex channel fading on the $m^{\text {th }}$ sub-carrier from the $p^{\text {th }}$ transmit antenna to the $q^{\text {th }}$ receive antenna, whose amplitude and phase are assumed to be Rayleigh distributed with $P_{c h}=E\left\{\left|H_{p, q, m}\right|^{2}\right\}=1$ and uniformly distributed in $[0,2 \pi]$, respectively. Moreover, the sub-carrier correlation coefficient between $H_{p, q, m_{0}}$ and $H_{p, q, m_{1}}$ is given by

$\rho_{m_{0}, m_{1}}=E\left\{H_{p, q, m_{0}}^{*} H_{p, q, m_{1}}\right\}=1 / \sqrt{1+\left(\Delta f / \Delta f_{c}\right)^{2}}$

where $\Delta f$ is the frequency separation between the $m_{0}^{\text {th }}$ and $m_{1}^{\text {th }}$ sub-carriers and $\Delta f_{c}$ is the coherence bandwidth of the channel. For different transmit or receive antennas, the channel fading is assumed to be independent to each other.

\section{Receiver}

After the FFT on the $\bar{q}^{\text {th }}$ receive antenna, the signals on the $\bar{m}^{\text {th }}$ sub-carrier is given by

$y_{\bar{q}, \bar{i}, \bar{m}}= \begin{cases}\sum_{p=0}^{n_{t}-1} H_{p, \bar{q}, \bar{m}} d_{p i l o t, p, \bar{m}} c_{N_{P}, \bar{i}}^{(p)}+\eta_{\bar{q}, \bar{i}, \bar{m}} & 0 \leq \bar{i} \leq N_{P}-1 \\ \sum_{p=0}^{n_{t}-1} H_{p, \bar{q}, \bar{m}} \sum_{k=0}^{K-1} d_{p, \bar{i}, \bar{m}, k} C_{\bar{i}, \bar{m}, k}+\eta_{\bar{q}, \bar{i}, \bar{m}} & N_{P} \leq \bar{i} \leq N_{d}-1\end{cases}$

where $\bar{q}=0, \cdots, n_{r}-1$, and $\eta_{\bar{q}, \bar{i}, \bar{m}}$ is the AWGN noise with zero mean and variance of $\sigma_{n}^{2}$. The first $N_{P}$ signals carry pilot symbols and are used for channel estimation. On the $\bar{m}$ th sub-carrier, the channel factor from the $\bar{p}$ th transmit antenna to the $\bar{q}^{\text {th }}$ receive antenna is given by

$\widetilde{H}_{\bar{p}, \bar{q}, \bar{m}}=\left(d_{p i l o t, \bar{p}}\right)^{-1}\left(\sum_{\bar{i}=0}^{N_{P}-1} y_{\bar{q}, \bar{i}, \bar{m}} c_{N_{P}, \bar{i}}^{(\bar{p})} / N_{P}\right)=H_{\bar{p}, \bar{q}, \bar{m}}+\nu_{\bar{p}, \bar{q}, \bar{m}}$

where $\nu_{\bar{p}, \bar{q}, \bar{m}}$ is the noise with zero mean and variance of $\sigma_{H}^{2}=\sigma_{n}^{2} /\left(\beta P_{d} N_{P}\right)$.

At the same time, on each sub-carrier, the data signals are processed by the time domain despreader to collect the useful signals in the time domain. Consider the data signals received on the $0^{\text {th }},\left(M / N_{F}\right)^{\text {th }}, \cdots$, and $\left(M-M / N_{F}\right)^{\text {th }}$ sub-carriers from the $\left(N_{P}\right)^{\text {th }}$ to the $\left(N_{P}+N_{T}-1\right)^{\text {th }}$ symbol duration, carrying the same unspread data symbol $d_{p, 0,0, k}$ (denoted as $d_{p, k}$ hereafter). Assuming that the desired data symbol is 2-D spread by $\left\{C_{N_{T}}^{(0)}, C_{N_{F}}^{(\bar{k})}\right\}$, the output of time domain despreader is given by

$$
\begin{array}{r}
\bar{y}_{\bar{q}, \bar{m}}=\frac{1}{N_{T}}{ }^{\sum_{\bar{i}=N_{P}}^{N_{P}+N_{T}-1} y_{\bar{q}, \bar{i}, \bar{m}} c_{N_{T},\left(\bar{i}-N_{P}\right) \% N_{T}}^{(0)}=H_{\bar{p}, \bar{q}, \bar{m}}\left(d_{\bar{p}, 0} c_{N_{F}, \bar{m}}^{(\bar{k})}\right)+\bar{\eta}_{\bar{q}, \bar{m}}} \\
+\underbrace{\sum_{\substack{p=0 \\
n_{t}-1}} H_{p, \bar{q}, \bar{m}}\left(\sum_{k=0}^{K_{C}} d_{p, k} c_{N_{F}, \bar{m}}^{(k)}\right)}_{M A I}+\underbrace{H_{\bar{p}, \bar{q}, \bar{m}}\left(\sum_{\substack{k=0 \\
k \neq \bar{k}}}^{K_{C}} d_{\bar{p}, k} c_{N_{F}, \bar{m}}^{(k)}\right)}_{M C I}
\end{array}
$$

$$
\text { process }
$$

antenna are recovered.

After the $0^{\text {th }}$ loop, a set of initial data decisions are obtained for all transmit antennas. In later loops, instead of ZF-SIC, MAI cancellation and maximum ratio combining (MRC) is carried out in the space domain to detect the signal transmitted on all antennas. Then, on each antenna, hybrid detection is employed to recover data symbols transmitted on each code channel.

\section{OPTIMAL POWER RATIO}

In practice, the total transmission power is limited. The power should be suitably assigned to the pilot and data channels. Assume that the transmission power on each antenna is fixed to $P_{t o t}=K M\left(N_{d}-N_{P}\right) P_{d}+M N_{P} \beta P_{d}$, then different power allocations are realized by changing the (5) values of $\beta$ or $P_{d}$. To obtain the optimal power allocation achieving the best system performance, an optimal $\beta$ or an optimal power ratio between the pilot channel and all data 
channels $\left(\beta_{\text {opt }} / K\right)$, equivalently, is needed.

In a MIMO system with ZF-SIC, the system performance is limited by the quality of the signals on the $0^{\text {th }}$ detected antenna, or the $0^{\text {th }}$ transmit antenna in this paper. Therefore, the proposed optimal power allocation scheme aims to provide the best performance for the $0^{\text {th }}$ antenna at the $0^{\text {th }}$ loop. As shown in Fig. 2, hybrid detection is carried out to collect the desired data from $N_{F}$ interleaved sub-carriers. Taking the MSE on each detected data symbol as a performance criterion, which can be approximated by [6]

$M S E=\left(P_{d}^{-1}+\left(\sigma^{2}\right)^{-1} \sum_{\bar{m}=0,\left(M / N_{F}\right), \cdots,\left(M-M / N_{F}\right)}\left|\hat{r}_{\bar{m}, 0,0}\right|^{2}\right)^{-1}$,

the optimal power ratio can be obtained by minimizing the normalized MSE, i.e., $M S E / P_{d}$.

First of all, $\sigma^{2}$ has been given in (7). Secondly, $\left|\hat{r}_{\bar{m}, 0,0}\right|^{2}$ follows a chi-square distribution with an expectation value of $E\left\{\left|\hat{r}_{\bar{m}, 0,0}\right|^{2}\right\}=\left(n_{r}-n_{t}+1\right)\left(P_{c h}+\sigma_{H}^{2}\right)$. Assuming that the frequency spacing between the $N_{F}$ interleaved sub-carriers are large and the frequency correlation between these subcarriers can be ignored, $\left|\hat{r}_{\bar{m}, 0,0}\right|^{2}$ are i.i.d. variables for $\bar{m}=0$, $M / N_{F}, \cdots, M-M / N_{F}$. The summation over $\left|\hat{r}_{\bar{m}, 0,0}\right|^{2}$ can be approximated by the expectation of $\left|\hat{r}_{m, 0,0}^{-}\right|^{2}$ as follows:

$\sum_{\bar{m}=0, \cdots,\left(M-M / N_{F}\right)}\left|\hat{r}_{\bar{m}, 0,0}\right|^{2} \approx N_{F}\left(n_{r}-n_{t}+1\right)\left(P_{c h}+\sigma_{n}^{2} / \beta P_{d} N_{P}\right)$.

As verified by simulations, the optimal power ratio obtained with this approximation works well for both small and large $N_{F}$. Moreover, assuming QPSK modulation, the average SNR per bit on each receive antenna is given by

$S N R_{b}=N P_{\text {tot }} /\left[2 K M\left(N_{d}-N_{P}\right) \sigma_{n}^{2}\right]$.

Substituting $\sigma^{2}$, (9) and (10) into $M S E / P_{d}$, the normalized MSE becomes a function of $\beta / K$. The optimal power ratio that achieves the maximum MSE can be obtained by setting the derivative $\partial\left(M S E / P_{d}\right) / \partial(\beta / K)$ to zero, resulting in

$$
\begin{aligned}
& \beta_{\text {opt }} / K=\left(N_{d}-N_{P}\right) /\left[N_{P}\left(\left(N_{d}-N_{P}\right)+A_{S N R}^{-1}\right)\right] \\
& \cdot\left\{\sqrt{n_{t}\left(N_{d}-N_{P}\right)+\left[n_{t}-\left(N_{d}-N_{P}\right)\right] A_{S N R}^{-1}}-A_{S N R}^{-1}\right\}
\end{aligned}
$$

where $A_{S N R}=2 P_{c h} S N R_{b}(K / N)$. When SNR is sufficiently large, (11) can be simplified to

$$
\lim _{S N R_{b} \rightarrow+\infty} \beta_{\text {opt }} / K=\sqrt{n_{t}\left(N_{d}-N_{P}\right) / N_{P}^{2}} \text {. }
$$

which suggests that the optimal $\beta_{\text {opt }} / K$ is mainly determined by the properties of channel estimation, represented by the spatial efficient $N_{P} / n_{t}$ and the time efficiency $N_{P} /\left(N_{d}-N_{P}\right)$.

\section{SimULATION RESULtS}

In the MIMO-OFCDM system, the number of transmit antennas and receive antennas are both four. The system bandwidth is $100 \mathrm{MHz}$ with $M=1024$ sub-carriers. The 2-D spreading scheme is set to $N=4 \times 16$. A full loaded system with $K=N$ data code channels is considered. Each packet is composed of $N_{d}=52$ OFCDM symbols, with the first $N_{P}$ symbols reserved as pilot symbols. Moreover, the coherence bandwidth $\Delta f_{c}$ is set to $1 \mathrm{MHz}$. At the receiver, a 4-loop joint iterative detection with 2-stage hybrid detection is employed.

The average BER is shown in Fig. 3 as a function of $\beta / K$ with different $N_{P}$. In general, it can be seen that when $\beta / K$ is small, the pilot channel has small power and the channel estimation quality is poor. Thus, the system performance degrades and the BER is high. When $\beta / K$ increases, the BER reduces as the quality of channel estimation improves. The BER reaches a minimum value for a particular value of $\beta / K$. Further increasing $\beta / K$ beyond that value increases BER due to too little power assigned to data channels. With $N_{P}=32,16,8$, and 4 , the minimum average BER is achieved when $\beta / K$ takes values around $0.3,0.8,1.5$ and 3.5, respectively. On the other hand, using (12), the analytical optimal power ratios can be calculated as $0.31,0.75,1.66$ and 3.46 , respectively. Clearly, the analytical results match well with the simulations.

Next, given $N_{P}=4$, the derived optimal power ratio is also verified in Fig. 4 for different $S N R_{b}, N_{F}$, no. of loops in the joint detection and antennas. It is shown that the system always achieves near optimum performance when $\beta / K$ takes values between 2.0 to 6.0 , which coincide with the derived $\beta_{\text {opt }} / K \approx 3.5$. Therefore, it has been verified by simulations that $\beta_{\text {opt }} / K$ is determined by the properties of channel estimation, while other system parameters have little influence on the its value.

\section{CONCLUSIONS}

The power allocation in MIMO-OFCDM systems has been investigated in this paper. A close form expression has been derived for the optimal power ratio $\left(\beta_{\text {opt }} / K\right)$ between the pilot and all data channels to achieve the best system performance. It has been verified by simulations that the derived $\beta_{\text {opt }} / K$ is accurate. $\beta_{\text {opt }} / K$ is mainly determined by the number of transmit antennas and the number of pilot and data symbols in one packet, while is not sensitive to other system parameters like SNR or $N_{F}$.

\section{ACKNOWLEDGMENT}

This work was supported by The University of Hong Kong Research Committee.

\section{REFERENCES}

[1] H. Atarashi, S. Abeta, and M. Sawahashi, "Broadband packet wireless access appropriate for high-speed and high-capacity throughput," IEEE VTC2001-Spring, pp. 566-570, May 2001.

[2] K. Higuchi, H. Kawai, N. Maeda and M. Sawahashi, "Adaptive selection of surviving symbol replica candidates based on maximum reliability in 
QRM-MLD for OFCDM MIMO multiplexing," in Proc. IEEE Globecom2004, pp. 2480-2486, Nov. 2004.

[3] Y. Q. Zhou and T. S. Ng, "Joint Iterative Detection for Multi-code MIMO-OFCDM Systems", in Proc. IEEE ICCSC 2008, pp. 434-438, May 26-28 2008.

[4] Y. Q. Zhou, J. Wang, T.S. Ng, K. Higuchi and M. Sawahashi, “OFCDM:
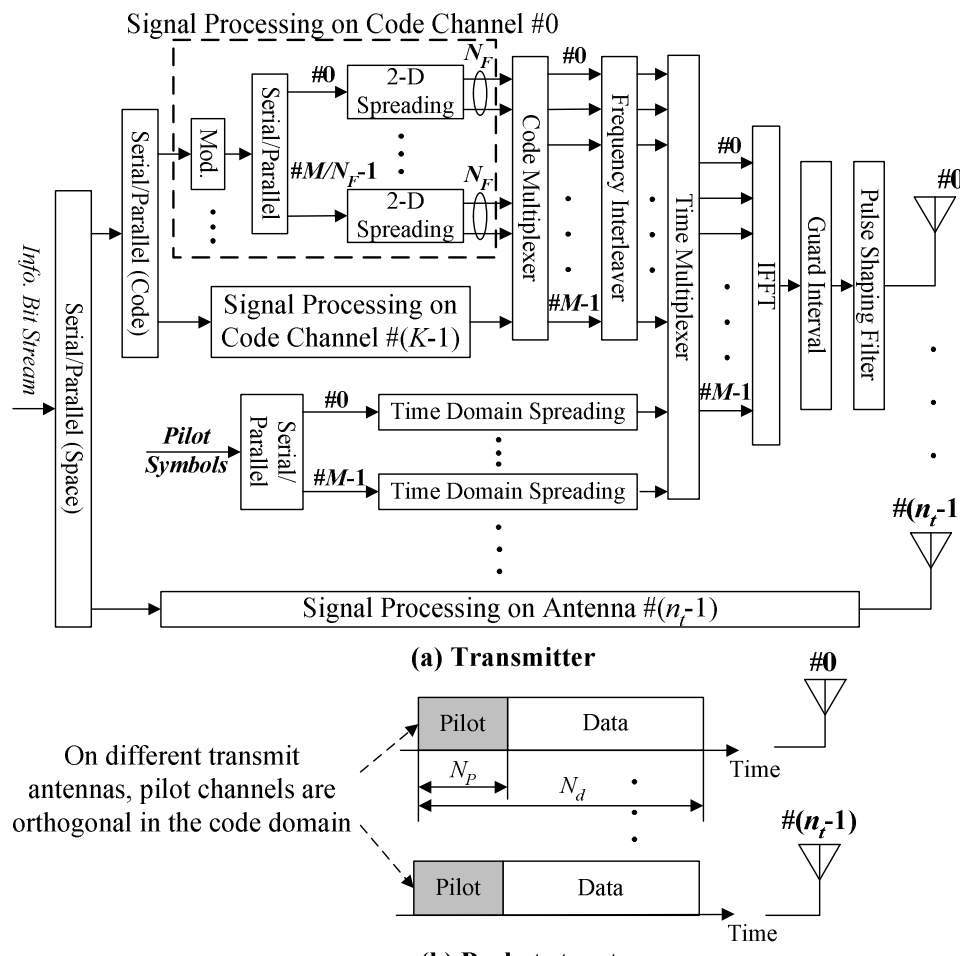

(b) Packet structure

Figure 1. Transmitter block diagram of the MIMO-OFCDM system

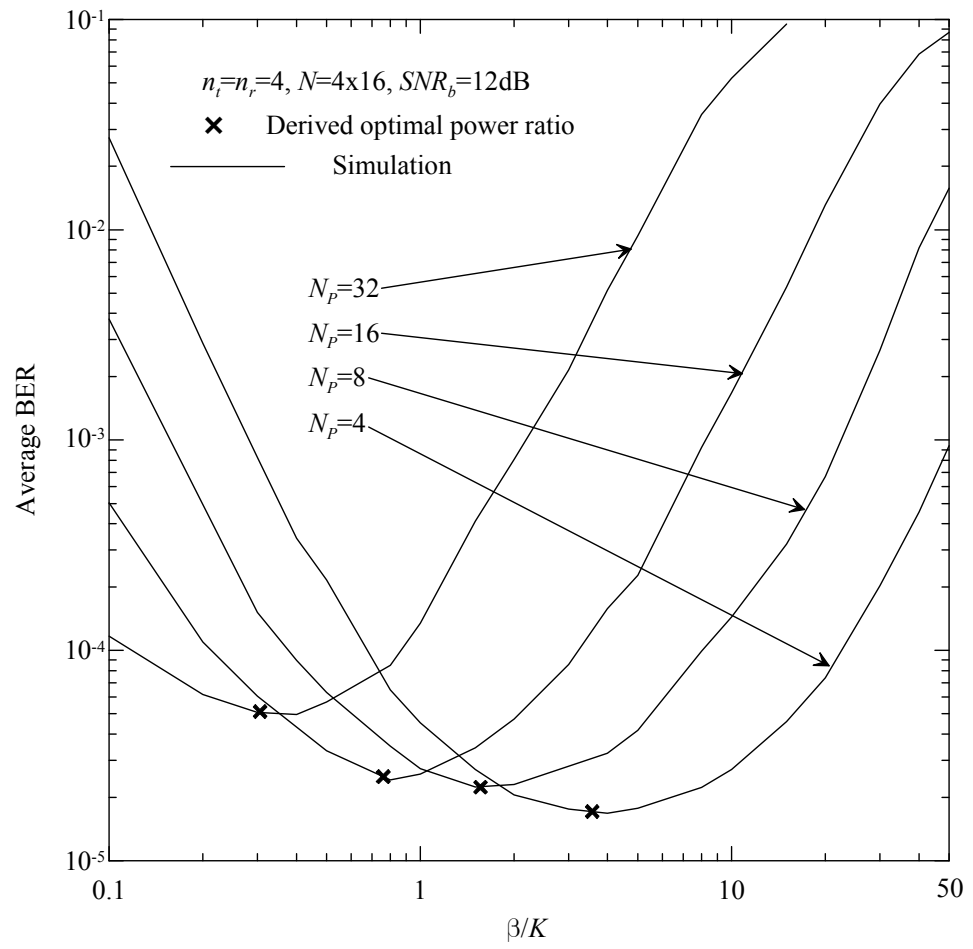

Figure 3. Verification of the optimal power ratio with different $N_{P}$ a promising broadband wireless access technique", IEEE Comm. Mag., vol. 46, pp. 39-49, March 2008.

[5] C. Shen, Y. Zhu, S. Zhou and J. Jiang, "On the performance of VBLAST with zero-forcing successive interference cancellation receiver", in Proc. IEEE Globecom2004, pp. 2818-2822, Dec. 2004.

[6] Sergio Verdú, "Multiuser Detection", Cambridge University Press, 1998.

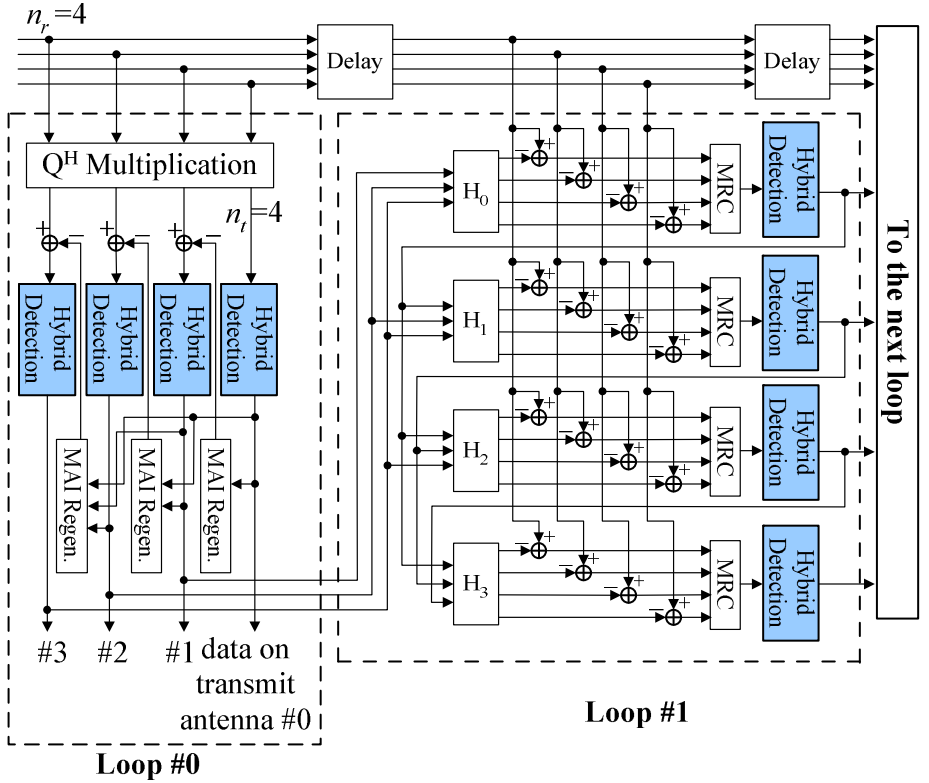

Figure 2. Example of joint iterative signal detection for $n_{t}=n_{r}=4$

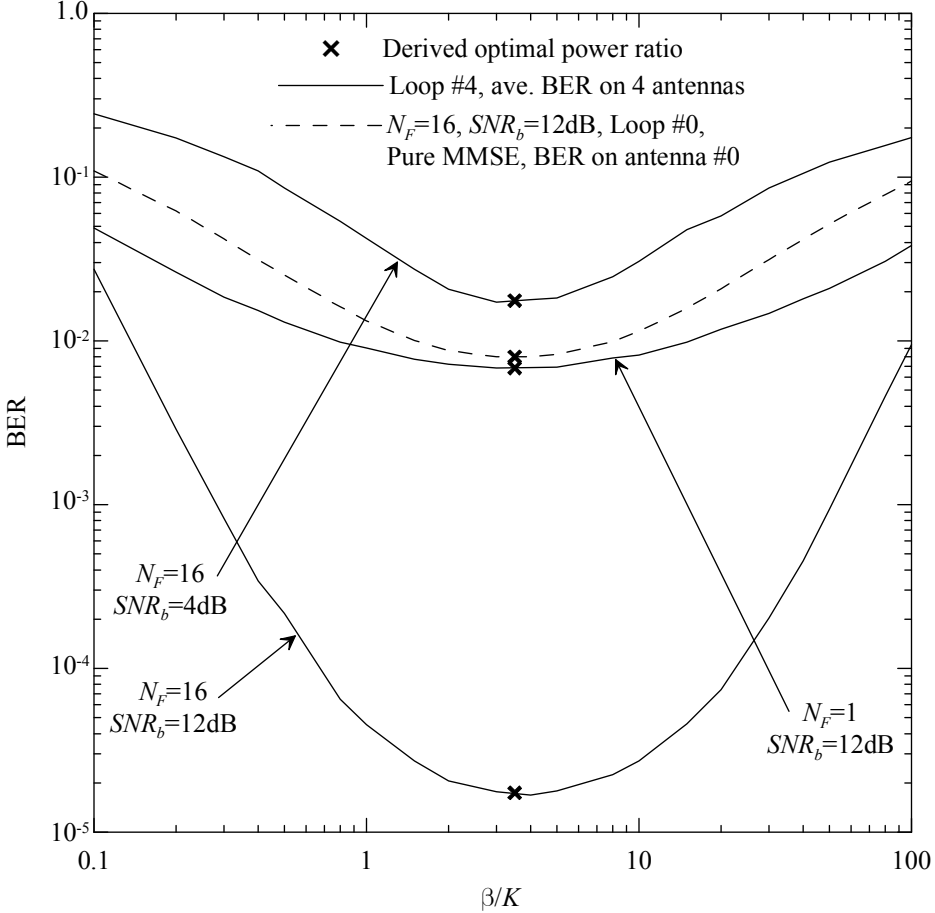

Figure 4. Verification of the optimal power ratio with different $S N R_{b}$ and $N_{F}$ 